Research Article

\title{
Trait Impulsivity Is Independent of Mild Cognitive Impairment in a Parkinson's Disease Cohort
}

\author{
Ashani Jeyadevan, ${ }^{1,2}$ Megan C. Bakeberg $\mathbb{D}^{2,3}$ Michelle Byrnes, ${ }^{2,3}$ Jade Kenna, ${ }^{2,3}$ \\ Soumya Ghosh, ${ }^{2,3}$ Rick Stell, ${ }^{2,3}$ Sue Walters, ${ }^{2,3}$ Tess Evans, ${ }^{4}$ Sarah McGregor, ${ }^{5}$ \\ Malcolm Horne, ${ }^{5,6}$ Frank L. Mastaglia, ${ }^{2}$ and Ryan S. Anderton (D) ${ }^{1,2,3,4}$ \\ ${ }^{1}$ School of Health Sciences, University of Notre Dame Australia, Fremantle, WA, Australia \\ ${ }^{2}$ Perron Institute for Neurological and Translational Science, Nedlands, WA, Australia \\ ${ }^{3}$ Centre for Neuromuscular and Neurological Disorders, University of Western Australia, Nedlands, WA, Australia \\ ${ }^{4}$ Institute of Health Research, University of Notre Dame Australia, Fremantle, WA, Australia \\ ${ }^{5}$ Florey Institute for Neuroscience and Mental Health, University of Melbourne, Parkville, Victoria 3010, Australia \\ ${ }^{6}$ Centre for Clinical Neurosciences and Neurological Research, St. Vincent's Hospital Melbourne, Fitzroy, Victoria 3065, Australia
}

Correspondence should be addressed to Ryan S. Anderton; ryan.anderton@nd.edu.au

Received 27 June 2019; Revised 7 September 2019; Accepted 12 September 2019; Published 1 October 2019

Guest Editor: Nicola Ray

Copyright (c) 2019 Ashani Jeyadevan et al. This is an open access article distributed under the Creative Commons Attribution License, which permits unrestricted use, distribution, and reproduction in any medium, provided the original work is properly cited.

\begin{abstract}
Introduction. Patients with Parkinson's disease (PD) commonly experience cognitive deficits and some also develop impulse control disorders (ICDs); however, the relationship between impulsivity and cognitive dysfunction remains unclear. This study investigated whether trait impulsivity associates with mild cognitive impairment (MCI), or is altered in a PD patient cohort with MCI. Methods. A total of 302 patients with idiopathic PD were recruited sequentially from three Australian Movement Disorder clinics. Based on cognitive scores, participants were divided into two groups, one defined as having mild cognitive impairment (PD-MCI; $n=113$ ) and the other with normal cognitive function (PD-C; $n=189$ ). Trait impulsivity was evaluated using the Barrett Impulsiveness Scale 11 (BIS-11). Total impulsivity scores, as well as subscale scores, were compared between PD-C and PD-MCI groups. Results. The PD-MCI cohort had significantly lower scores in all cognitive domains, and mirrored expected clinical differences in medication, motor symptoms, and disease duration, when compared to the PD-C cohort. Self-reported impulsivity was not significantly different between groups, nor was there a difference within first-order subscale scores: attention $(p=0.137)$, cognitive instability $(p=0.787)$, self-control $(p=0.503)$, cognitive complexity $(p=0.157)$, motor impulsivity ( $p=0.559)$, or perseverance $(p=0.734)$ between the PD-MCI and PD-C groups. Conclusions. These findings suggest that impulsive traits and behaviors are independent of changes in cognitive state and are not altered in PD patients with mild cognitive impairment.
\end{abstract}

\section{Introduction}

Parkinson's disease (PD) is a complex neurodegenerative disorder in which the cardinal motor symptoms are accompanied by a variety of nonmotor symptoms (NMS) including olfactory, autonomic, psychiatric, and cognitive dysfunction. Prodromal cognitive deficits are of particular interest, offering potential insight into disease progression and early diagnosis, as well as targets for disease-modifying therapies. Cross-sectional studies have observed that approximately $30 \%$ of people with $\mathrm{PD}(\mathrm{PwP})$ have dementia $[1,2]$. Furthermore, a 20-year longitudinal Australian study revealed that over $80 \%$ of $\mathrm{PwP}$ develop increasing cognitive impairment as the disease progresses and eventually become demented [3].

There has also been increasing awareness of the occurrence of abnormal impulsive-compulsive behaviors in $\mathrm{PD}$ patients as the disease progresses, particularly those 
treated with dopamine agonist drugs, which have been found to occur in as many as $\sim 46 \%$ of patients followed over a 5-year period [4-6]. These have been collectively termed impulse control disorders (ICDs), include pathological gambling, shopping, eating, hoarding, and hypersexuality, as well as compulsive use of dopaminergic medications ("dopamine dysregulation syndrome") $[7,8]$, and are all regarded as compulsive reward-seeking forms of behavior. They confer heightened levels of distress for both patients and carers, as well as having serious implications for quality of life [9]. Heightened trait impulsivity is present in a subset of PD patients, particularly males [10], and is considered a risk factor for the development of ICDs.

The neural substrate of ICDs and increased impulsivity in $\mathrm{PD}$ is thought to involve dysregulation in mesolimbic and mesocortical networks, and changes in dopamine receptor (D2 and D3) binding in the ventral striatum [5, 11], but specific neuropathological correlates have not been identified. However, based on the disease staging studies by Braak et al., it might be envisaged that changes in impulsivity could correlate with Stage V, in which the Lewy pathology extends to the mesolimbic cortex, and could precede, or overlap with, the development of impaired cognition, as there is further extension to neocortical areas in Stage VI of the disease [12].

Although impulsivity and cognitive impairment frequently coexist in $\mathrm{PD}$, it is unclear whether there are any interactive effects between them during the course of the disease. A link between impulsive traits and low cognitive scores has been observed in other cohorts, such as children with attention-deficit hyperactive disorder (ADHD) [13]. In addition, preliminary evidence suggests that, in PD, cognitive characteristics such as poor executive abilities, as well as poor action control and response inhibition, and certain personality traits such as negative affectivity and high premorbid levels of novelty seeking, may have impact on impulsivity and be risk factors for the development of ICDs $[6,11,14]$. However, relatively little is known about the link between cognitive abilities and behavioral changes in patients with PD, and there have been conflicting findings in the literature [15-17]. In particular, the relationship between subclinical impulsiveness and mild cognitive impairment (PD-MCI) in patients without a diagnosed ICD remains unclear, as previous studies have focused on cohorts with diagnosed behavioral disorders.

Accordingly, the aim of this cross-sectional study was to evaluate the relationship between MCI and subclinical impulsivity as measured using the Barratt Impulsiveness Scale 11 (BIS-11) in an Australian multicenter PD patient cohort. More specifically, we questioned whether in PD patients with MCI, there are changes in attentional and motor impulsiveness, and in measures of cognitive complexity and instability.

\section{Methodology}

2.1. Participants. A total of 302 patients were recruited sequentially into the Australian Parkinson's Disease Registry (APDR) from Movement Disorder Clinics at the Perron
Institute in Perth, St. Vincent's Hospital in Melbourne, and Royal North Shore Hospital in Sydney. In all cases, the diagnosis of idiopathic PD was confirmed in accordance with the UK Brain Bank criteria prior to inclusion in the study. At the time of all assessments, patient response to medication was at optimum levels ("ON" period). Written informed consent was obtained from all participants, in accordance with the National Health and Medical Research Council guidelines. Patients who were unable to complete the cognitive and impulsivity protocols or had a diagnosed ICD were excluded from this study.

2.2. Demographic and Clinical Assessment. Patient demographic and clinical information was collected, including age, gender, date of diagnosis, motor symptom severity, smoking status, dopaminergic medications, and deep brain stimulation (DBS) history. For each patient, the total daily intake of all dopaminergic medications was converted to a levodopa equivalent dose (LED), as described elsewhere [18]. Part III of the Movement Disorders Society Unified Parkinson's Disease Rating Scale (MDS-UPDRS III) was employed to evaluate the severity of motor symptoms and was conducted by a clinician or trained research nurse. Lastly, patient quality of life data were collected using the self-assessed, 39-item Parkinson's Disease Questionnaire (PDQ-39) [19].

2.3. Assessment of Impulsivity. The Barratt Impulsiveness Scale 11 (BIS-11) is a self-report validated evaluation and assesses impulsivity as a multifaceted entity [20]. Secondorder attentional impulsiveness is described as an inability to concentrate and can be categorised into first-order subscales for attention and cognitive instability. Motor impulsiveness is the tendency to act without thinking, with first-order motor and perseverance scales. Lastly, nonplanning impulsiveness is an inability to plan for the future, within which there are first-order subscales for cognitive complexity and self-control. The BIS-11 is entirely self-rated, with each item marked on a four-point scale, giving patients a score between 30 and 120. As the scoring scheme is reversed in some questions, each question was marked individually to ensure that higher BIS-11 scores gave a true indication of heightened impulsiveness.

2.4. Assessment of Global Cognition. Global cognition impairments were assessed using Addenbrooke's Cognitive Examination-Revised (ACE-R) [21], which has been used previously to determine cognitive impairment in $\mathrm{PD}$ patient cohorts [22, 23]. The ACE-R evaluated five prominent cognitive domains, with a maximum total score of 100 points: orientation and attention (18 points), memory (26 points), verbal fluency (14 points), language ( 26 points), and visuospatial (16 points) abilities. In all cognitive domains, lower scores represent poorer cognitive abilities. On the basis of the ACE-R scores, patients were allocated to either an MCI group (PD-MCI) or a cognitively normal group 
(PD-C), according to a verified total ACE-R score cut-off of 88.5 (sensitivity 0.68 and specificity 0.91 ) [24].

2.5. Statistical Analysis. All data were analysed using IBMSPSS (v. 25, IBM Corporation) and presented as mean \pm standard deviation unless otherwise stated. For comparisons between PD-C and MCI groups, nonparametric Mann-Whitney $U$ test was performed. Models were corrected for covariates that were demonstrated to significantly differ between PD-C and PDMCI cohorts. A significant nominal $p$ value of $<0.05$ was employed.

\section{Results}

3.1. Cognitive Characteristics of PD-C and PD-MCI Groups. The cohort was initially divided into two groups based on the presence or absence of mild cognitive impairments (PDMCI and PD-C, respectively), as determined by ACE-R scores. ACE-R subdomains were compared between cognitive groups, revealing significant differences in each subdomain. Throughout attention and orientation $(p<0.001)$, memory $(p<0.001)$, fluency $(p<0.001)$, language $(p<0.001)$, and visuospatial $(p<0.001)$ domains, significant differences were present between the PD-C and PD-MCI subgroups (Table 1).

3.2. Cohort Demographics and Clinical Differences between $P D-C$ and $P D-M C I$ Cohorts. Table 2 summarizes clinical and demographic characteristics of the complete patient cohort $(n=302)$, the PD-MCI subgroup, and the PD-C subgroup. Patients in the PD-MCI group were more likely to be older $(63.2 \pm 9.48$ vs. $66.4 \pm 8.51, p=0.003)$, have a longer disease duration $(7.39 \pm 5.09$ vs. $9.75 \pm 6.10, p<0.001)$, be male $(\mathrm{OR}=4.24, p=0.039)$, to have more severe motor symptoms $(17.7 \pm 11.0$ vs. $23.6 \pm 15.4, p=0.002)$, and a poorer quality of life $(26.9 \pm 18.8$ vs. $40.9 \pm 26.4, p<0.001)$ than those in the $\mathrm{PD}-\mathrm{C}$ cohort. In terms of therapeutic interventions, the PD-MCI group were more likely to be using DBS treatment $(\mathrm{OR}=4.72, p=0.030)$ and have a higher LED $(765 \pm 571$ vs. $970 \pm 620, p<0.001)$ than those with normal cognitive function. As such, further analysis of the relationship between impulsivity scores and cognitive status included age at assessment, gender, disease duration, LED, and DBS as covariates.

3.3. Impulsivity Scores in PD-C and PD-MCI Groups. Total BIS-11 scores of all patients ranged from 30 to 102 , with an interquartile range of 12 points. Patients with PD$\mathrm{MCI}$, compared to those who were PD-C, scored higher on total impulsivity measures and multiple subscales of impulsivity; however, no differences reached statistical significance (Table 3 ). BIS-11 subscales were only analysed in corrected comparisons where significance was seen in naïve pairwise comparison.

Specifically, individuals within the PD-MCI group had higher total BIS-11 scores, although not statistically significant $(59.5 \pm 8.77$ vs. $60.2 \pm 10.2, p=0.318)$. Secondorder attentional subscale findings reflected total BIS-11 scores, with differences between groups remaining minimal with no significance noted $(15.3 \pm 3.18$ vs. $15.4 \pm 3.80$, $p=0.704)$. Within this attentional subscale, first-order attentional subscale scores also saw no significant differences $(p=0.137)$, whilst cognitive instability scores were significantly different $(p=0.019)$. However, in fully corrected models controlled for confounding factors, cognitive stability was no longer significant in effect $(p=0.787)$. Subsequent analysis of second-order nonplanning scores and motor scores indicated no differences in mean scores $(23.8 \pm 4.81$ vs. $23.8 \pm 5.67, p=0.175 ; 21.0 \pm 3.26$ vs. $21.0 \pm 2.90, p=0.737)$, mirrored by insignificant differences in first-order subscales (Table 3).

\section{Discussion}

Cognitive dysfunction is an important nonmotor manifestation of $\mathrm{PD}$, which increases in frequency and severity as the disease progresses, and can range from mild impairment in one or more cognitive domains, to outright dementia. People with PD are twice as likely to develop MCI and six times more likely to become demented when compared to age-matched controls [25]. Furthermore, the onset of PDMCI has been recognized as a predictive factor for other debilitating symptoms such as sleep problems, depression, hallucinations, and ICDs [26]. It has been suggested that cognitive impairment may be involved in the development of ICDs $[15,16,27]$, and it has been proposed that the two disorders may share a common underlying neurobiological substrate [8].

While it has previously been suggested that there may be a link between cognitive impairment and trait impulsivity, which is thought to underlie the development of ICDs, we were unable to demonstrate such an association in the present study using the ACE-R cognitive screening protocol. We did not find any significant differences in attentional, nonplanning, or motor impulsivity BIS-11 measures between a PD-MCI group and a cognitively normal PD group (PD-C), when controlling for other confounding factors. The findings of this study therefore suggest that the presence of PD-MCI in PwP does not align with higher levels of subclinical impulsivity, nor is there evidence for an inverse relationship between impaired cognition and impulsivity, or any indication of a change in any specific impulsivity traits in the cognitively impaired PD group. However, the study did not investigate the influence of domain-specific MCI, which may relate to impulsivity subscales.

Contention exists in the literature surrounding the relationship between cognition and ICDs, with some studies in PD cohorts with diagnosed ICDs reporting no differences in cognitive abilities and others reporting a better level of functioning in some tasks assessing cognition [17, 28]. Moreover, conflicting conclusions have been reached in regard to which specific cognitive functions relate to ICDs. In the current study, the PD-MCI group performed significantly lower in all cognitive domains, and it is possible that impairments in some of these domains could lead to 
TABle 1: Differences in ACE-R subdomain scores in the PD-C and PD-MCI groups.

\begin{tabular}{|c|c|c|c|c|}
\hline & All subjects $(n=302)$ & PD-C $(n=189)$ & PD-MCI $(n=113)$ & Arm comparison \\
\hline ACE-R total & $88.02(11.3)$ & $94.4(3.09)$ & $77.4(12.1)$ & $t=14.6(p<0.001)$ \\
\hline Attention and orientation & $17.3(1.56)$ & $17.9(0.364)$ & $16.4(2.18)$ & $t=10.4(p<0.001)$ \\
\hline Memory & $21.8(4.63)$ & $24.3(1.79)$ & $17.6(4.88)$ & $t=12.3(p<0.001)$ \\
\hline Fluency & $9.23(3.71)$ & $11.3(2.28)$ & $5.79(3.04)$ & $t=12.2(p<0.001)$ \\
\hline Language & $24.8(1.99)$ & $25.4(1.02)$ & $23.9(2.75)$ & $t=5.85(p<0.001)$ \\
\hline Visuospatial & $14.7(2.44)$ & $15.5(1.00)$ & $13.5(3.42)$ & $t=5.84(p<0.001)$ \\
\hline
\end{tabular}

TABle 2: Clinical and demographic characteristics of cohort, when grouped by cognitive status.

\begin{tabular}{|c|c|c|c|c|}
\hline & All subjects $(n=302)$ & PD-C $(n=189)$ & PD-MCI $(n=113)$ & Arm comparison \\
\hline Age of assessment (years) & $64.4(9.20)$ & $63.2(9.48)$ & $66.4(8.51)$ & $t=-2.95(p=0.003)$ \\
\hline Gender: male (\%) & $186(61.6)$ & $108(57.1)$ & $78(69.0)$ & $\mathrm{OR}=4.28(p=0.039)$ \\
\hline Disease duration (years) & $8.27(5.60)$ & $7.39(5.09)$ & $9.75(6.10)$ & $t=-3.57(p<0.001)$ \\
\hline MDS-UPDRS III score & $19.9(13.1)$ & $17.7(11.0)$ & $23.6(15.4)$ & $t=-3.17(p=0.002)$ \\
\hline Total levodopa equivalent dosage (mg/day) & $841(597)$ & $765(571)$ & $970(620)$ & $t=-3.046(p<0.001)$ \\
\hline Deep brain stimulation treatment (\%) & $32(11.0)$ & $14(4.8)$ & $18(15.9)$ & $\mathrm{OR}=4.72(p=0.030)$ \\
\hline Parkinson's Disease Questionnaire (PDQ-39) & $32.5(23.1)$ & $26.9(18.8)$ & $40.9(26.4)$ & $t=-4.785(p<0.001)$ \\
\hline
\end{tabular}

TABLE 3: Total BIS-11 and first- and second-order scores in the overall cohort and PD-C and PD-MCI groups.

\begin{tabular}{|c|c|c|c|c|c|}
\hline & $\begin{array}{l}\text { All subjects } \\
\quad(n=302)\end{array}$ & $\begin{array}{c}\text { PD-C } \\
(n=189)\end{array}$ & $\begin{array}{l}\text { PD-MCI } \\
(n=113)\end{array}$ & $\begin{array}{l}\text { Naïve pairwise } \\
\text { comparison }\end{array}$ & $\begin{array}{l}\text { Covariate corrected } \\
\text { comparison }\end{array}$ \\
\hline BIS-11 total score & $59.7(9.33)$ & $59.5(8.77)$ & $60.2(10.2)$ & $p=0.318$ & NS \\
\hline Second-order attentional & $15.3(3.42)$ & $15.3(3.18)$ & $15.4(3.80)$ & $p=0.704$ & NS \\
\hline First-order attentional & $10.1(2.66)$ & $9.96(2.53)$ & $10.4(2.56)$ & $p=0.137$ & NS \\
\hline First-order cognitive instability & $5.21(1.58)$ & $5.34(1.49)$ & $4.98(1.70)$ & $p=0.019$ & $p=0.787$ \\
\hline Second-order nonplanning & $23.4(5.15)$ & $23.8(4.81)$ & $23.8(5.67)$ & $p=0.175$ & NS \\
\hline First-order self-control & $12.07(3.56)$ & $12.0(3.29)$ & $12.2(3.73)$ & $p=0.503$ & NS \\
\hline First-order cognitive complexity & $11.3(2.58)$ & $11.1(2.47)$ & $11.57(2.74)$ & $p=0.157$ & NS \\
\hline Second-order motor & $21.0(3.51)$ & $21.1(3.26)$ & $21.0(2.90)$ & $p=0.737$ & NS \\
\hline First-order motor & $13.6(2.98)$ & $13.7(2.77)$ & $13.5(3.33)$ & $p=0.559$ & NS \\
\hline First-order perseverance & $7.46(1.71)$ & $7.43(1.63)$ & $7.50(1.85)$ & $p=0.734$ & NS \\
\hline
\end{tabular}

${ }^{*} \mathrm{NS}=$ not significant.

higher impulsivity, whilst impairments in other domains could mitigate other aspects of trait impulsivity. The failure to find any such associations in this study may reflect limitations of the BIS-11 self-reporting scale, and further studies employing more sensitive instruments for trait impulsivity and more comprehensive cognitive testing protocols would therefore be worthwhile to explore this possibility further.

No study has yet demonstrated a relationship between impulsivity and cognition in a cohort of PwP, though an association has been established in other populations. The BIS-11 scale quantifies an individual's perception of various behaviors, thoughts, and actions that are associated with impulsivity [20]. It has been suggested that individuals with lower cognitive abilities may be less likely to comprehend the consequences of reporting their impulsive events, and are therefore more likely to disclose impulserelated behaviors and feelings, and to self-report high impulsivity. This phenomenon has been reported in prison inmates [29], and in children with learning difficulties [30] and ADHD [13]. Alternatively, many individuals with higher cognitive abilities are thought to under-report impulsive behaviors due to embarrassment, or because they are fearful of potential consequences in disclosing impulsive behaviors. The extent to which these potential limitations of the self-reported BIS-11 scale may apply to the PD patient cohort is uncertain, although it is possible that individuals who have MCI may be less able to objectively identify problematic behaviors, and that participants in a research study may have different attitudes to reporting impulsive traits.

\section{Limitations}

A number of other limitations warrant consideration when interpreting the findings of the present study. Firstly, the self-report nature of the BIS-11 assessment is likely to introduce a degree of variability and bias in responses. As patients may be reluctant to report impulsive tendencies, this may limit the reliability of the results of the study. While more objective behavioral measures to assess situational "state" impulsivity and to screen for specific personality traits were not employed in this study, it is important that these aspects be addressed in future studies. In regard to 
cognition, the PD-C and PD-MCI groupings were established using predefined cut-off scores for global MCI, and the study did not investigate the influence of domain-specific MCI, which may relate to impulsivity subscales, or relation between executive function and nonplanning impulsivity. Furthermore, possible overlap exists between certain items in the BIS-11 and ACE-R scales: e.g., the "attentional impulsiveness" subscale in the BIS-11 and the "attention and orientation" domain in the ACE-R. Moreover, the crosssectional nature of the study precludes any possible conclusions of a causal relationship, or lack thereof, between deficits in specific cognitive domains and impulsiveness.

\section{Conclusion}

The present results have established that subtle shifts in cognitive circuitry, as related to PD-MCI, do not necessarily associate with individual variability in trait impulsivity. Absence of any obvious relationship between the complex multidimensional constructs of impulsivity and cognition suggests that the underlying neurobiology may not relate, at least at a subclinical level. This prompts a need to reconsider the underpinnings of impulsivity in PD as a separate construct to cognition, in order to better characterize and treat symptoms experienced by patients.

\section{Data Availability}

The data that support the findings of this study are available from the corresponding author upon reasonable request.

\section{Conflicts of Interest}

The authors declare that they have no conflicts of interest.

\section{Acknowledgments}

This study was funded by grants/research support from the Federal Cooperative Research Centre for Mental Health, the Perron Institute for Neurological and Translational Science, and the University of Notre Dame Australia. This research was carried out while MCB was in receipt of a Richard Walter Gibbon Medical Research Scholarship and an Australian Government Research Training Program Scholarship at the University of Western Australia.

\section{References}

[1] D. Aarsland, K. Brønnick, and T. Fladby, "Mild cognitive impairment in Parkinson's disease," Current Neurology and Neuroscience Reports, vol. 11, no. 4, pp. 371-378, 2011.

[2] B. Dubois, D. Burn, C. Goetz et al., "Diagnostic procedures for Parkinson's disease dementia: recommendations from the movement disorder society task force," Movement Disorders, vol. 22, no. 16, pp. 2314-2324, 2007.

[3] M. A. Hely, W. G. J. Reid, M. A. Adena, G. M. Halliday, and J. G. L. Morris, "The Sydney multicenter study of Parkinson's disease: the inevitability of dementia at 20 years," Movement Disorders, vol. 23, no. 6, pp. 837-844, 2008.
[4] J.-C. Corvol, F. Artaud, F. Cormier-Dequaire et al., "Longitudinal analysis of impulse control disorders in Parkinson disease," Neurology, vol. 91, no. 3, pp. e189-e201, 2018.

[5] D. Latella, M. G. Maggio, G. Maresca et al., "Impulse control disorders in Parkinson's disease: a systematic review on risk factors and pathophysiology," Journal of the Neurological Sciences, vol. 398, pp. 101-106, 2019.

[6] C. Nombela, T. Rittman, T. W. Robbins, and J. B. Rowe, "Multiple modes of impulsivity in Parkinson's disease," PLoS One, vol. 9, no. 1, Article ID e85747, 2014.

[7] D. Weintraub, J. Koester, M. N. Potenza et al., "Impulse control disorders in Parkinson disease: a cross-sectional study of 3090 patients," Archives of Neurology, vol. 67, no. 5, pp. 589-595, 2010.

[8] D. Weintraub, A. D. Siderowf, M. N. Potenza et al., "Association of dopamine agonist use with impulse control disorders in Parkinson disease," Archives of Neurology, vol. 63, no. 7, pp. 969-973, 2006.

[9] Y.-S. Oh, J. E. Lee, P. H. Lee, and J.-S. Kim, "Neuropsychiatric symptoms in Parkinson's disease dementia are associated with increased caregiver burden," Journal of Movement Disorders, vol. 8, no. 1, pp. 26-32, 2015.

[10] M. Riley, M. Bakeberg, M. Byrnes et al., "Demographic and clinical predictors of trait impulsivity in Parkinson's disease patients," Parkinson's Disease, vol. 2018, Article ID 9472120, 7 pages, 2018.

[11] D. Weintraub, "Impulse control disorders in Parkinson's disease: a 20-year odyssey," Movement Disorders, vol. 34, no. 4, pp. 447-452, 2019.

[12] H. Braak, K. D. Tredici, U. Rüb, R. A. I. de Vos, E. N. H. Jansen Steur, and E. Braak, "Staging of brain pathology related to sporadic Parkinson's disease," Neurobiology of Aging, vol. 24, no. 2, pp. 197-211, 2003.

[13] R. Gupta and B. R. Kar, "Specific cognitive deficits in ADHD: a diagnostic concern in differential diagnosis," Journal of Child and Family Studies, vol. 19, no. 6, pp. 778-786, 2010.

[14] G. Santangelo, F. Piscopo, P. Barone, and C. Vitale, "Personality in Parkinson's disease: clinical, behavioural and cognitive correlates," Journal of the Neurological Sciences, vol. 374 , pp. 17-25, 2017.

[15] R. Biundo, P. Formento-Dojot, S. Facchini et al., "Brain volume changes in Parkinson's disease and their relationship with cognitive and behavioural abnormalities," Journal of the Neurological Sciences, vol. 310, no. 1-2, pp. 64-69, 2011.

[16] A. Djamshidian, A. Jha, S. S. O’Sullivan et al., "Risk and learning in impulsive and nonimpulsive patients with Parkinson's disease," Movement Disorders, vol. 25, no. 13, pp. 2203-2210, 2010.

[17] A. Djamshidian, S. S. O’Sullivan, A. Lees, and B. B. Averbeck, "Stroop test performance in impulsive and non impulsive patients with Parkinson's disease," Parkinsonism \& Related Disorders, vol. 17, no. 3, pp. 212-214, 2011.

[18] C. L. Tomlinson, R. Stowe, S. Patel, C. Rick, R. Gray, and C. E. Clarke, "Systematic review of levodopa dose equivalency reporting in Parkinson's disease," Movement Disorders, vol. 25, no. 15, pp. 2649-2653, 2010.

[19] L. C. S. Tan, N. Luo, M. Nazri, S. C. Li, and J. Thumboo, "Validity and reliability of the PDQ-39 and the PDQ-8 in English-speaking Parkinson's disease patients in Singapore," Parkinsonism \& Related Disorders, vol. 10, no. 8, pp. 493-499, 2004.

[20] J. H. Patton, M. S. Stanford, and E. S. Barratt, "Factor structure of the Barratt impulsiveness scale," Journal of Clinical Psychology, vol. 51, no. 6, pp. 768-774, 1995. 
[21] E. Mioshi, K. Dawson, J. Mitchell, R. Arnold, and J. R. Hodges, "The Addenbrooke's Cognitive Examination Revised (ACER): a brief cognitive test battery for dementia screening," International Journal of Geriatric Psychiatry, vol. 21, no. 11, pp. 1078-1085, 2006.

[22] M. C. Bakeberg, A. Jefferson, M. Riley et al., "Elevated serum homocysteine levels have differential gender-specific associations with motor and cognitive states in Parkinson's disease," Parkinson's Disease, vol. 2019, Article ID 3124295, 8 pages, 2019.

[23] T. Evans, A. Jefferson, M. Byrnes et al., "Extended "Timed up and Go" assessment as a clinical indicator of cognitive state in Parkinson's disease," Journal of the Neurological Sciences, vol. 375, pp. 86-91, 2017.

[24] D. Berankova, E. Janousova, M. Mrackova et al., "Addenbrooke's cognitive examination and individual domain cut-off scores for discriminating between different cognitive subtypes of Parkinson's disease," Parkinson's Disease, vol. 2015, Article ID 579417, 7 pages, 2015.

[25] D. Aarsland, K. Andersen, J. P. Larsen, A. Lolk, H. Nielsen, and P. Kragh-Sorensen, "Risk of dementia in Parkinson's disease: a community-based, prospective study," Neurology, vol. 56, no. 6, pp. 730-736, 2001.

[26] K. F. Pedersen, J. P. Larsen, O.-B. Tysnes, and G. Alves, "Prognosis of mild cognitive impairment in early Parkinson disease," JAMA Neurology, vol. 70, no. 5, pp. 580-586, 2013.

[27] V. Voon, B. Reynolds, C. Brezing et al., "Impulsive choice and response in dopamine agonist-related impulse control behaviors," Psychopharmacology, vol. 207, no. 4, pp. 645-659, 2010.

[28] C. Siri, R. Cilia, D. Gaspari et al., "Cognitive status of patients with Parkinson's disease and pathological gambling," Journal of Neurology, vol. 257, no. 2, pp. 247-252, 2010.

[29] P. Snoyman and B. Aicken, "Self-reported impulsivity in male offenders with low cognitive ability in New South Wales prisons," Psychology, Crime \& Law, vol. 17, no. 2, pp. 151-164, 2011.

[30] R. Donfrancesco, D. Mugnaini, and A. Dell'Uomo, "Cognitive impulsivity in specific learning disabilities," European Child \& Adolescent Psychiatry, vol. 14, no. 5, pp. 270-275, 2005. 


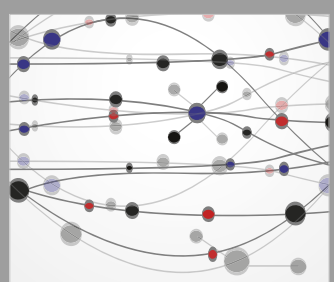

The Scientific World Journal
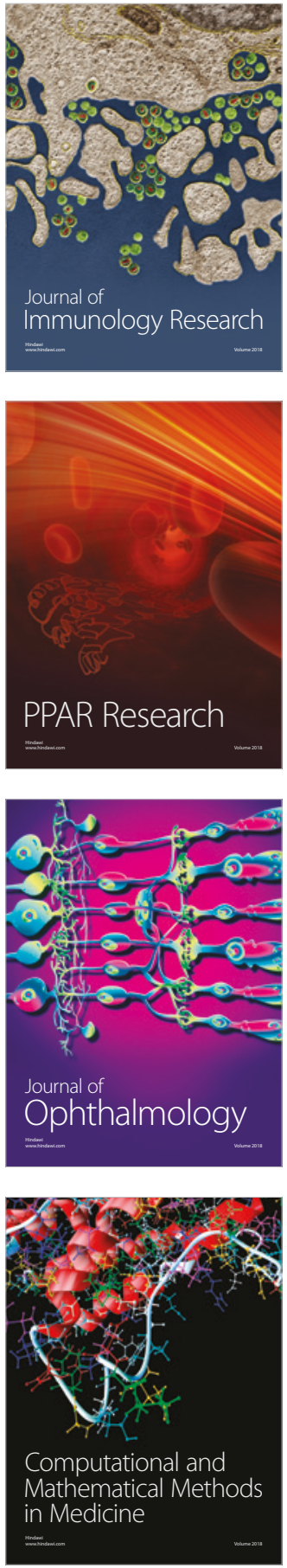

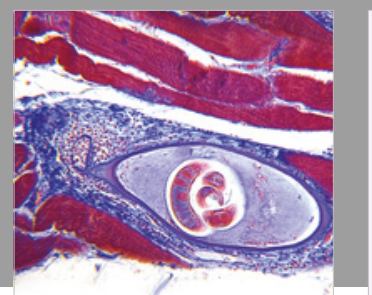

Gastroenterology Research and Practice

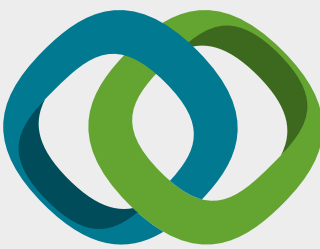

\section{Hindawi}

Submit your manuscripts at

www.hindawi.com
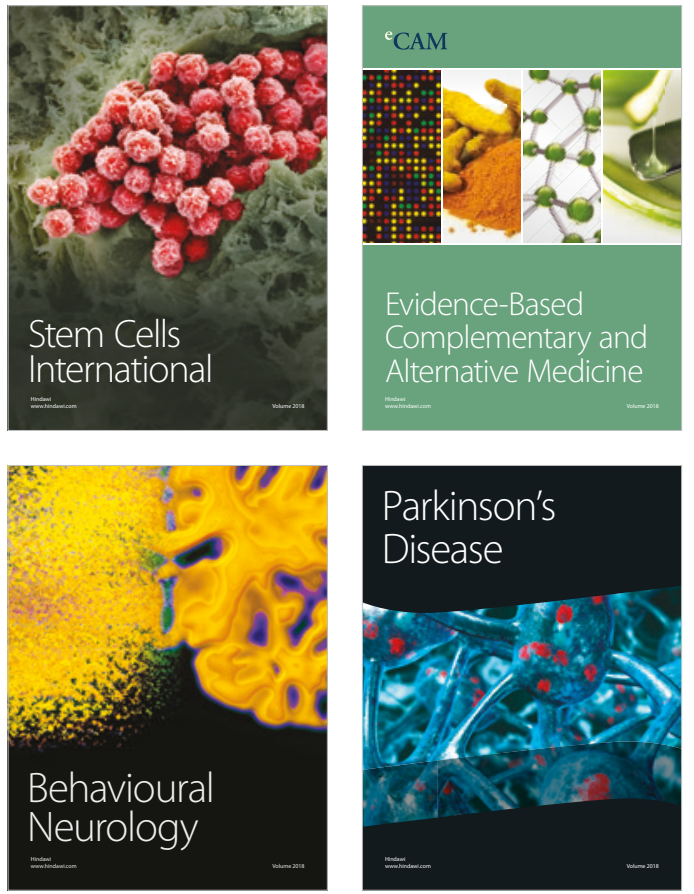

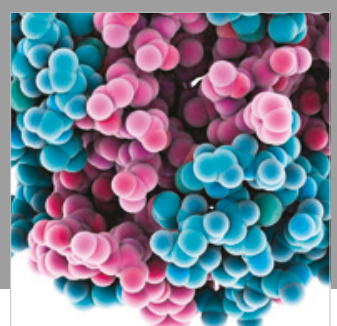

ournal of

Diabetes Research

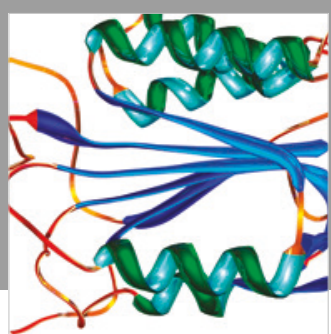

Disease Markers
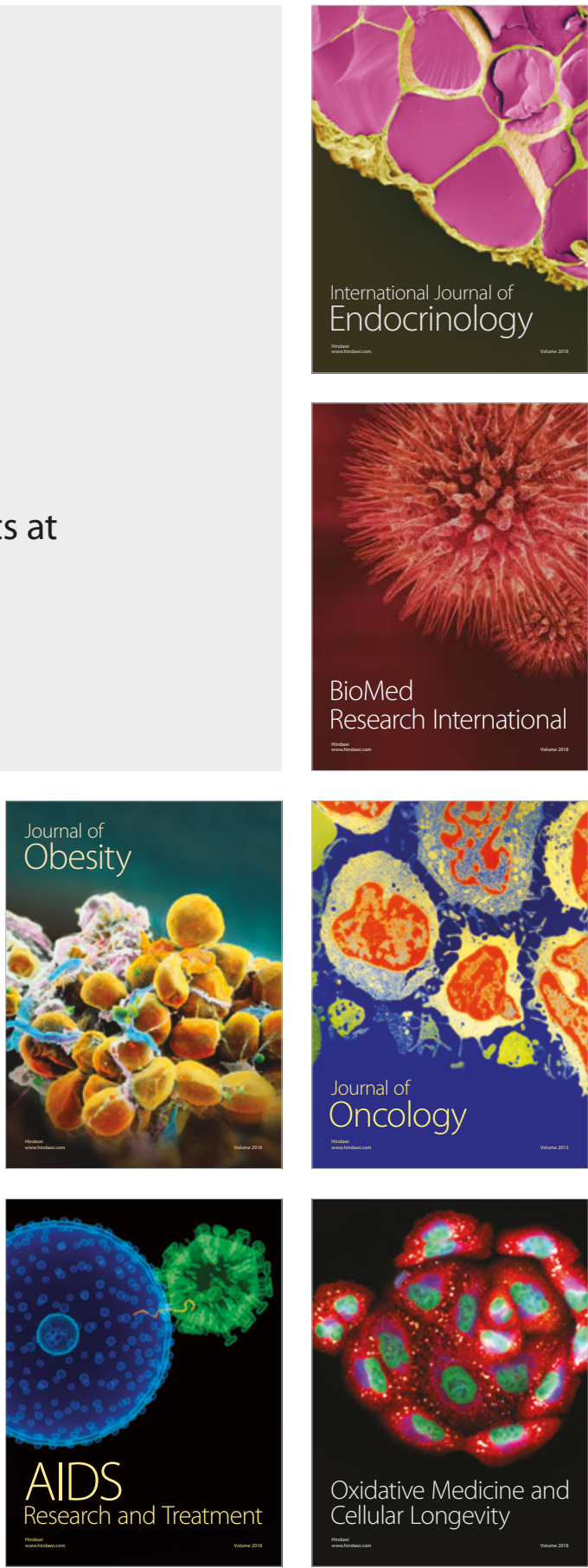